\title{
Erratum to: Quantum Sense and Nonsense
}

\section{Erratum to: \\ J. Bricmont, Quantum Sense and Nonsense, https://doi.org/10.1007/978-3-319-65271-9}

In the original version of the book, the sentence "Copernicus Books is a brand of Springer" has to be included at the bottom of copyright page after "Printed on acid-free paper" line in frontmatter. The erratum book has been updated with the change. 